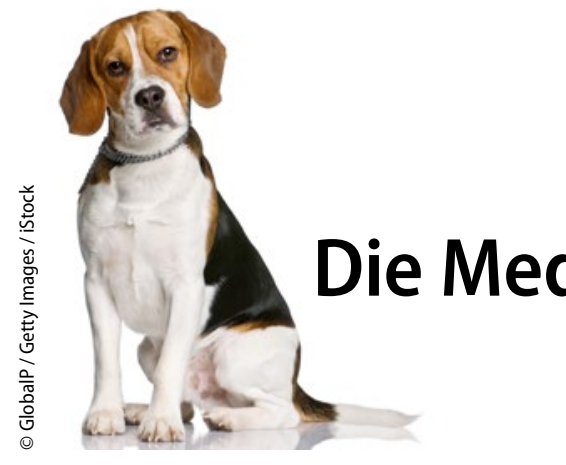

Zukunftsforscher schätzen, dass $80 \%$ der medizinischen Fragestellungen künftig ohne Arzt rein digital gelöst werden können. Auch in der Medizin soll also die natürliche Intelligenz durch die künstliche ersetzt werden. Doch da könnten sich die Auguren verrechnet haben.

Die Verfechter der künstlichen Intelligenz in der Medizin wurden nämlich kürzlich durch eine Meldung aufgeschreckt, die nicht mit ihrem Weltbild der Zukunft kompatibel sein dürfte. Die Rede ist von Hunden, genauer gesagt von mexikanischen Beagles, die sich bei der Tumorsuche als höchst zuverlässige Diagnostiker qualifizieren konnten. Durch das Beschnüffeln von Damenbinden konnten diese, wie die ÄrzteZeitung berichtet, ein Zervixkarzinom mit erstaunlicher Sicherheit detektieren. Die Spezifität und Sensitivität dieses kynologisch-gynäkologischen Verfahrens lag bei über $90 \%$, also höher als beim PapTest oder einer HPV-Testung. Das soll ein Computer erst einmal nachmachen!

Ähnlich hoch war die Trefferquote bei Hunden, die aus dem Urin ein Prostatakarzinom erschnüffeln konnten. Und auch bei der Frühdiagnostik des Bronchialkarzinoms, bei der die apparative Diagnostik bisher versagt hat, könn- te der Hund nach ersten Erfahrungen durch das Beschnüffeln der Ausatemluft wertvolle Dienste leisten. Somit spricht manches dafür, dass der Arzt zumindest beim Tumorscreening in absehbarer Zeit von einem Hund ersetzt wird. Dies mag manchem Kollegen mit einer narzistisch geprägten Unersetzlichkeitsphantasie zwar weh tun, aber auch hier gilt die normative Kraft des Faktischen. Natürliche bzw. tierische Intelligenz versus elektronische! Dies dürfte die neue Fragestellung sein. Im Moment spricht zumindest vieles dafür, dass Kollege „Dr. Hund“ schneller Realität sein könnte als Kollege „Dr. Computer“.

Dr. Peter Stiefelhagen

\title{
Arztumfrage
}

\section{Das Richtige zu tun ist wichtiger als Geld}

\section{Man muss sich schon berufen fühlen, um als Arzt glücklich zu werden. Geld allein trägt nach den Ergebnissen einer Umfrage wenig zum Wohlbefinden bei.}

_ Zu diesen Schlüssen kommen Gesundheitsforscher von der Universität in Omaha (Nebraska, USA), nachdem sie in einer repräsentativen Umfrage knapp 1.300 US-Ärzte aller Fachrichtungen konsultiert haben.

Alle Teilnehmer, darunter $40 \%$ Hausärzte, erhielten einen Bogen mit 38 Fragen zu Karriere, Verdienst, Zufriedenheit und Engagement. Etwas mehr als ein Drittel verdiente jährlich zwischen 100.000 und 200.000 Dollar, ein Viertel lag bei 200.000-300.000 Dollar und rund ein Fünftel darüber. Knapp ein
Fünftel musste mit weniger als 100.000 Dollar klarkommen.

Der Aussage „Arzt zu sein ist eine Berufung"stimmten $88 \%$ der Befragten zu, ebenso hoch war die Zufriedenheit mit dem Beruf (86\%) und dem Leben allgemein (87\%). Dennoch fühlten sich $46 \%$ ausgebrannt oder gaben zu, mit der Zeit abgestumpft zu sein.



Fachärzte waren etwas unzufriedener als Allgemeinärzte. Die Spitzenverdiener zeigten die höchste Zufriedenheit mit ihrer beruflichen Entwicklung, attestierten ihrem Leben tendenziell jedoch weniger Sinn als das Fünftel mit dem geringsten Verdienst.

Als wichtigster Wohlfühlfaktor erwies sich die Zeit, die mit sinnvollen und befriedigenden Tätigkeiten verbracht wurde. Lag diese Zeit bei weniger als zweieinhalb Stunden am Tag, waren die Ärzte rund fünffach häufiger unzufrieden als Ärzte mit mehr als fünf Stunden - und zwar unabhängig vom Verdienst. Die höchste Zufriedenheit zeigten Ärzte, die viele Langzeitbeziehungen zu ihren Patienten pflegten.

- Tak HJ et al. J Gen Intern Med, online 7. Februar 2017 